\title{
O PROCESSO DE ENFERMAGEM EM UMA UNIDADE DE QUEIMADOS: ANÁLISE E REFORMULAÇÃO FUNDAMENTADAS NA PEDAGOGIA DA PROBLEMATIZAÇÃO*
}

\author{
Lídia Aparecida Rossi ** \\ Maria Auxiliadora Trevisan ***
}

\begin{abstract}
O presente estudo constitui na elaboração e aplicação de um plano de atividade educativa para enfermeiras de uma Unidade de Queimados, com o objetivo de analisar e reformular a prática do processo de enfermagem nesta unidade.

O "Esquema do Arco" proposto por Charles MAGUEREZ 8 foi empregado como guia, norteando a escolha em e a aplicação das atividades de ensino-aprendizagem implantados neste plano de atividade educativa.

Concluímos que esse esquema pedagógico possibilita a ocorrência de transformações na prática do processo de enfermagem e que esta nova realidade já nos mostra a necessidade de outras transformações.
\end{abstract}

UNITERMOS: processo de enfermagem, educação em enfermagem, assistência de enfermagem.

\section{INTRODUÇÃO}

Para HORTA ${ }^{7}$, o processo de enfermagem compreende seis fases interrelacionadas: histórico, diagnóstico de enfermagem, plano assistencial, prescrição, evolução e prognóstico de enfermagem. Dessas fases, o histórico, a prescrição e a evolução de enfermagem são as que têm sido implementadas com maior freqüência.

*Resumo de Dissertação de Mestrado apresenta à Escola de Enfermagem de Ribeirão Preto da Universidade de São Paulo

**Mestre pela Escola de Enfermagem de Ribeirão Preto da Universidade de São Paulo. Diretora do Serviço de Enfermagem de Centro Cirúrgico e Central de Material da Unidade de Emergência do Hospital das Clínicas da Faculdade de Medicina de Ribeirão Preto da Universidade de São Paulo

${ }^{* * *}$ Professora Titular e Chefe do Departamento de Enfermagem Geral e Especializada da Escola de Enfermagem de Ribeirão Preto, Centro Colaborador da Organização Mundial da Saúde para o desenvolvimento da pesquisa em Enfermagem. 
Na Unidade de Queimados do Hospital das Clínicas da Faculdade de Medicina de Ribeirão Preto da Universidade de São Paulo (HCFMRP-USP), onde desenvolvemos este estudo, essas três fases têm sido aplicadas desde 1987.

Na nossa prática, muitas vezes o que observamos é que essa metodologia de assistência tornou-se, para o enfermeiro, mais uma obrigação burocrática, e prescrição de enfermagem, uma lista de rotinas a serem cumpridas. Não existe uma inter-relação entre o histórico, a prescrição e a evolução de enfermagem.

Assim como tem sido praticada, essa metodologia de assistência não contribuído para garantir um cuidado individualizado.

Muitos são os fatores que tem favorecido a sua não implementação ou simplificação. Destacamos a resistência do próprio enfermeiro, que desde a graduação percebe o processo de enfermagem como uma atividade burocrática ${ }^{9}$. Acreditamos que essa percepção pode estar relacionada à falta de exemplos práticos bem sucedidos, ou como afirma $\mathrm{SOUZA}^{18}$, à forma como o ensino teórico e prático do processo de enfermagem tem ocorrido nos cursos de graduação, não despertando nos alunos a compreensão e a consciência do seu valor para a assistência de enfermagem. Supomos que, em muitos casos, o ensino dessa metodologia de assistência não tem levado o educando a desenvolver habilidades para a solução de problemas e também não tem garantido a compreensão da estrutura do processo de enfermagem.

Neste estudo, entendemos que a estrutura do processo de enfermagem compreende cinco passos inter-relacionados: coleta de dados, diagnóstico, planejamento, implementação e avaliação.

Numa análise das propostas de $\mathrm{HORTA}^{7}$, e com base paciente queimado, ROSSI \& DALRI ${ }^{13}$ elaboraram um modelo de processo de enfermagem compreendendo cinco fases: coleta de dados, diagnóstico, plano assistencial, prescrição e evolução incluindo aqui o prognóstico de enfermagem, que se aproxima da estrutura do processo de enfermagem. Essas autoras elaboraram um instrumento para a coleta de dados considerando a classificação de necessidades adotada por HORTA ${ }^{7}$. Dessa forma, os dados objetivos e subjetivos são coletados obedecendo uma hierarquia de necessidades: psicobiológicas, psicossociais e psicoespirituais.

A fase de Diagnóstico, como proposta por HORTA ${ }^{7}$, foi considerada, por essas autoras como pouco operacional, não oferecendo uma base precisa para realização das etapas seguintes. ROSSI \& DALRI ${ }^{13}$ elaboraram uma proposta de adaptação da Taxionomia I de diagnóstico de enfermagem proposta pela North American Nursing Diagnois Association (NANDA), junto ao modelo de HORTA ${ }^{7}$.

Uma vez estabelecido o Diagnóstico, a fase seguinte, o planejamento, deve estar claramente delineada compreendendo: julgamento de prioridades, estabelecimento e desenvolvimento de objetivos e prescrição de enfermagem.

Na proposta de HORTA ${ }^{7}$, o passo seguinte ao Diagnóstico é a elaboração do plano assistencial. Na visão de ROSSI \& DALRI ${ }^{13}$, o plano assistencial proposto por essa autora, repetiria a prescrição de enfermagem. Dessa forma, optarem nessa fase, pela determinação dos objetivos a serem alcançados com a implementação do plano. 
A prescrição de enfermagem na proposta de ROSSI \& DALRI ${ }^{13}$, seria realizada a partir da determinação das prioridades e objetivos estabelecidos no plano assistencial.

A evolução de enfermagem é uma avaliação da assistência de enfermagem que deverá refletir as condições do cliente possibilitando a elaboração de um perfil do seu desempenho quanto ao alcance dos objetivos propostos ${ }^{13}$.

Considerando as implicações decorrentes da aplicação do processo de enfermagem de forma simplificada e a importância da compreensão da estrutura dessa metodologia de assistência, aplicamos um plano de atividade educativa fundamento do "Esquema do Arco" de Charles MAGUEREZ ${ }^{8}$ e nas propostas de ROSSI \& DALRI ${ }^{13}$, na Unidade de Queimados do HCFMRP-USP (U.Q.).

Abordaremos a seguir, o referencial teórico que fundamentou esse plano de atividade educativa.

\section{REFERENCIAL TEÓRICO}

\section{A EDUCAÇÃO RENOVADA OU PROGRESSISTA}

No início deste século, surgiu uma nova forma de compreensão do processo educacional: a Educação Renovada ou Progressista.

A Escola Nova tinha com fundamento para a aprendizagem, a atividade ${ }^{11}$.

Para DEWEY², considerando o grande mestre que influenciou a educação na primeira metade do século $X X$, a educação deveria desenvolver o pensamento reflexivo do educando e melhorar sua capacidade de resolver problemas.

Para DEWEY², o pensamento reflexivo é desencadeado por uma situação embaraçosa, perturbadora ou confusa. Essa situação é chamada de pré-reflexiva: é a origem da questão, apresenta o problema a ser resolvido. Na situação final, a dúvida foi dissipada; a situação é pós-reflexiva. Esses são os limites da reflexão.

As idéias de DEWEY ${ }^{2}$ tornaram-se conhecidas em todo o mundo e contribuíram para muitas outras que se sucederam na educação.

Como conseqüência da posição filosófica de $D^{2} E E^{2}$ em relação ao pensamento reflexivo, surgiu no inicio deste século o método de resolução de problemas.

Esse método tem sido descrito por CARVALHO ${ }^{1}, \mathrm{NÉRICl}^{10}$ e VILARINHO ${ }^{14}$, através de cinco etapas:

1. Definição e eliminação do problema;

2. Coleta, classificação e análise crítica dos dados;

3. Formulação de hipótese;

4. Seleção de hipóteses;

5. Verificação de hipóteses. 


\section{O ESQUEMA PEDAGÓGICO DE CHARLES MAGUEREZ}

Em 1970, com base nas etapas do método de solução de problemas, CHARLEZ MANGUEREZ propôs um esquema pedagógico para ser utilizado na assistência técnica ao agricultor, o "Esquema do Arco".

MANGUEREZ ${ }^{8}$ e DIAS BORDENAVE \& PEREIRA ${ }^{3}$ apresentam o "Esquema do Arco" em cinco fases:

$1^{\circ}$ Observação da realidade: Para MANGUEREZ ${ }^{8}$, esta fase consisti na observação de todos os dados da realidade e na seleção dos aspectos característicos a serem considerados na solução das questões levantadas.

$2^{\circ}$ Construção de uma maquete: consisti na elaboração de um modelo reduzido da realidade, orientado pela escolha dos aspectos característicos mais importantes para a solução do problema: os pontos-chave.

$3^{\circ}$ Esta fase foi chamada por MANGUEREZ ${ }^{8}$ de "discussão deste esquema". DIAS BORDANAVE \& PEREIRA ${ }^{3}$ chamaram esta fase de Teorização, considerando que nessa etapa é introduzida a discussão teórica do assunto que poderá confirmar, acrescentar, e/ou modificar os aspectos ressaltados na segunda fase.

$4^{\circ}$ MANGUREZ ${ }^{8}$ chamou esta fase de "execução de uma maquete", optamos por chamá-la de Hipótese de Solução assim como apresentada por DIAS BORDENAVE \& PEREIRA ${ }^{3}$.Consiste numa confrontação entre as discussões realizadas nas fases anteriores. Assim, as hipóteses de solução são confrontadas com os parâmetros da maquete do problema.

$5^{\circ}$ Execução Efetiva representa a adoção de uma solução e sua aplicação prática junto à realidade.

Para MANGUEREZ $Z^{8}$ este esquema pedagógico "satisfaz os cânones da aprendizagem, pois vai do concreto ao abstrato, para retornar ao concreto".

O "Esquema do Arco", apresentado na Figura 1, é entendido por MANGUEREZ ${ }^{8}$, como uma etapa de Instrução. Esta etapa é seguida por outras: Aplicação e Acompanhamento. Na etapa de aplicação, o instrutor deverá participar da execução, fazendo ensaios de aplicação e as correções quando necessárias. A etapa de Acompanhamento é uma continuação da aplicação, sendo assim, um controle da execução com as correções eventuais e informações práticas que forem necessárias.

Entendemos que nas etapas - Aplicação e Acompanhamento devem ser utilizados o mesmo esquema, que deverá ser realizado considerando a situação problema do momento, ou seja, "a nova realidade". Desse modo, as etapas subseqüentes. (Aplicação e Acompanhamento) só serão diferentes da fase da Instrução, considerando que deverá existir uma nova realidade e, assim, outros problemas deverão ser resolvidos com base no mesmo esquema pedagógico: "Uma seqüência de Arcos". 


\section{FIGURA 1: REPRESENTAÇÃO DO "ESQUEMA DOA ARCO" SEGUNDO MAGUEREZ ${ }^{8}$}

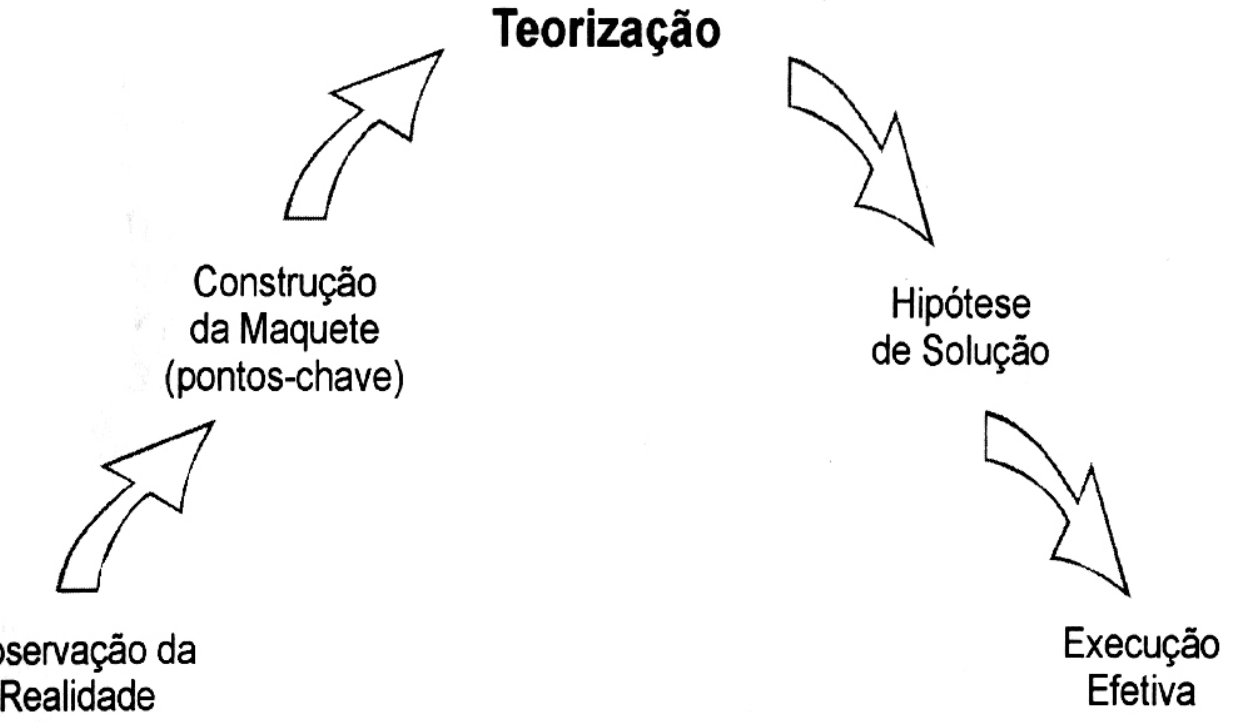

RE A L I D A D E

Fonte: DIAZ BORDENVE,J.; PEREIRA, A.M.

Estratégias de ensino-aprendizagem.

12. ed. Petrópolis, Vozes, 1991. p. 49

\section{OBJETIVOS DO ESTUDO}

\section{Objetivo Geral}

Analisar e reformular a prática de processo de enfermagem na Unidade de Queimados do HCFMRP-USP.

Para tanto, foram estabelecidas etapas metodológicas, com base mo "Esquema do Arco", de forma que este estudo levasse os sujeitos a:

- refletir sobre o significado de ser enfermeiros;

- refletir sobre a prática do processo de enfermagem na U.Q.;

- identificar os componentes do processo de enfermagem, 
- identificar os determinantes do avanço e do atraso do processo de enfermagem na U.Q;

- refletir sobre a importância e finalidade dos componentes do processo de enfermagem;

- elaborar e aplicar propostas para a reformulação da prática do processo de enfermagem na U.Q.

\section{A UNIDADE DE QUEIMADOS DO HOSPITAL DAS CLÍNICAS DA FACULDADE DE MEDICINA DE RIBEIRÃO DA UNIVERSIDADE DE SÃO PAULO, FRENTE À PROPOSTA DE ANÁLISE E REFORMULAÇÃO DA PRÁTICA DO PROCESSO DE ENFERMAGEM}

A Unidade de Queimados, onde desenvolvemos este estudo, é uma das seções do Hospital das Clínicas da Faculdade de Medicina de Ribeirão Preto da Universidade de São Paulo. Desde o início de suas atividades os enfermeiros prescreviam as ações de enfermagem. Essa prescrição, que estava vinculada à uma avaliação prévia do paciente, era registrada na folha de evolução de enfermagem, logo abaixo do registro da primeira avaliação feita pelo enfermeiro no período da manhã. Estava restrita aos aspectos físicos do tratamento ao paciente.

O quadro do pessoal, inicialmente suficiente para as atividades previstas para a Unidade, logo deixou de sê-lo. As internações ultrapassaram o número de leitos, sendo que até 1985 a taxa de ocupação girava em torno de $120 \%$.

Somava-se a essa situação o fato de que os enfermeiros da Unidade de Queimados eram constantemente deslocados para outras Unidades, sendo o oposto também ocorria. Quando enfermeiros de outros locais eram escalados para Unidade de Queimados, a prescrição de enfermagem nem sempre era realizada.

Desse modo, a prescrição de enfermagem que em grande parte, se constituía numa lista de procedimentos rotineiros a serem checados, logo deixou de ser feita.

A partir de 1987, o número de leitos foi ampliado de cinco para dez, o quadro de pessoal foi revisto e os enfermeiros passaram a ser exclusivo da Unidade de Queimados. O planejamento da assistência foi retomado e o processo de enfermagem passou a ser aplicado em três fases: histórico, prescrição e a evolução de enfermagem.

Na prática o histórico de enfermagem nem sempre era aplicado e a prescrição de enfermagem era feita diariamente, mas desvinculada dos problemas, que muitas vezes, quando explicitados na evolução de enfermagem, não encontravam a prescrição correspondente. Foram feitas modificações no instrumento de coleta de dados, mas ainda, assim a mesma era efetuada por poucos enfermeiros.

Em 1991, ROSSI \& DALRI ${ }^{13}$, enfermeiras da Unidade de Queimados, fizeram uma análise quanto aos fatores que dificultavam ou facilitavam a reformulação 
da prática do processo de enfermagem nessa Unidade. A partir das conclusões dessas autoras, entendemos que seria possível o desenvolvimento de um processo de mudança que pudesse levar à reformulação da prática do processo de enfermagem. Para o desenvolvimento de qualquer atividade que pretendesse a modificação essa prática, consideramos importante conhecer o envolvimento dos enfermeiros com 0 processo de enfermagem. Assim, passamos a realizar reuniões informais com o grupo de enfermeiros com o objetivo de discutir essas questões e levantar informações que pudessem subsidiar o planejamento de um processo de mudança.

Nessas reuniões o grupo demonstrou interesse pela continuidade do processo de enfermagem e concluiu que um curso seria importante para resgatar algumas questões. Desse modo, desenvolvemos um plano de atividades que apresentaremos a seguir.

\section{PLANO DE ATIVIDADE EDUCATIVA}

\section{Apresentação}

Para o alcance dos objetivos deste estudo, desenvolvemos um plano e atividade educativa fundamentado nas propostas de MAGUEREZ $^{8}$ e DIAS BORDENAVE \& PEREIRA ${ }^{3}$.

Fundamentamos o plano de atividade educativa, proposto neste estudo no "Esquema do Arco". Como sugerem DIAS BORDENAVE \& PEREIRA ${ }^{3}$, esse esquema foi utilizado como "uma linha pedagógica central que nos orientou na escolha de técnicas educativas e dos recursos multissensoriais".

Aplicamos esse plano a um grupo composto por enfermeiros da Unidade de Queimados do HCFMRP-USP, sendo: 01 diretor de serviço, 01 enfermeiro-chefe, 01 enfermeiro-encarregado, 06 enfermeiros.

De acordo com as propostas de MAGUEREZ ${ }^{8}$ essa atividade educativa compreendeu dois momentos:

Instrução: abrangeu a fase de execução do plano de atividade educativa.

Aplicação e Acompanhamento: essa fase dependia da anterior e compreendeu a aplicação e acompanhamento das propostas elaboradas durante a execução do plano.

Destacamos que nesses dois momentos, os dados foram coletados através da técnica de observação participante e registrados através do registro cursivo com papel e lápis, pelo coordenador e por um enfermeiro voluntário membro do grupo e através dos registros em quadros feitos pelo grupo durante o desenvolvimento da fase de instrução.

Os dados obtidos foram comparados e transcritos em um único registro pelo coordenador e submetidos a aprovação do enfermeiro voluntário. Nessa transcrição 
excluímos os diálogos que se repetiam ou fugiam completamente ao assunto e os que, por estarem incompletos, não permitiam interpretações.

\section{Descrição e análise das fases de instrução e aplicação e acompanhamento}

As considerações a seguir complementam o desenvolvimento da fase de Instrução e da fase de Aplicação e Acompanhamento respeitando a seqüência em que foram desenvolvidas.

\subsection{Fase de instrução}

Essa fase abrangeu três unidades. Cada unidade compreendeu diferentes atividades de ensino-aprendizagem. Como sugerem DIAS BORDENAVE \& PEREIRA ${ }^{3}$, através dessas atividades criamos situações que possibilitaram a ocorrência de experiências de aprendizagem que provocaram, no grupo, as mudanças desejadas.

\subsubsection{Unidade I - Análise da prática do processo de enfermagem na Unidade de Queimados}

Essa unidade compreendeu três atividades de ensino-aprendizagem: (I-1)

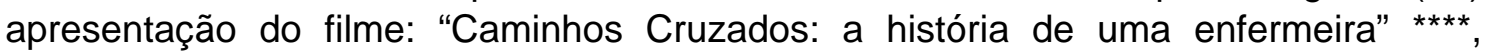
seguida de uma discussão em pequenos grupos; (I-2) aplicação da Técnica Tempestade de Idéias; (I-3) análise e discussão de registros de enfermeiros em dois prontuários de pacientes internados na Unidade de Queimados.

As atividades de ensino-aprendizagem desenvolvidas nesta unidade possibilitaram o desenvolvimento das duas primeiras fases do "Esquema do Arco"; a "Observação da Realidade" e o "Levantamento dos Pontos-chave".

Num primeiro momento dessa fase de observação da realidade, a utilização do filme permitiu que alguns aspectos da realidade fossem trazidos ao local de aula estimulando as discussões nas quais foram abordadas questões relacionadas ao papel do enfermeiro, que apresentamos a seguir:

- existem satisfações relacionadas a: cuidar, melhora do paciente, aproveitamento de aptidões no grupo, oportunidade de adquirir conhecimentos e atitudes cordiais dos pacientes;

${ }^{* \star \star \star}$ Caminhos Cruzados: a história de uma enfermeira Dart nele, Siamar, 27 min., son., color. (Filme) 
- existem insatisfações relacionadas a: salário, sofrimento e agressividade dos pacientes, relacionamento médico-enfermeiro e horário de trabalho;

- os enfermeiros entendem como seu papel: a administração do cuidado direto integral do paciente, ser competente e ter compromisso com a profissão.

Consideramos essas questões como pontos-chave desta primeira abordagem da realidade.

Uma reflexão sobre a opção pela enfermagem, sobre o significado de ser enfermeiro e sobre as situações que interferem no desempenho do seu papel, deve anteceder as discussões sobre o processo de enfermagem. Entendemos que a prática dessa metodologia de assistência implica em que o enfermeiro assuma a responsabilidade pelo planejamento da assistência.

Na nossa prática, participamos de reuniões que grande parte dos enfermeiros assume como responsabilidade apenas o cumprimento das ações prescritas pelo médico.

Nossa interpretação sobre os depoimentos obtidos nas reuniões realizadas antes do planejamento dessa atividade e após a apresentação do filme, é que os enfermeiros entendem com sua responsabilidade o cuidado e consideram necessário dar continuidade e modificar a prática do processo de enfermagem nesta unidade.

Com a aplicação da Técnica Tempestade de Idéias, essas reflexões tomaram outra direção. Essa direção foi proposta quando delimitamos o assunto: assistência de enfermagem e processo de enfermagem.

A Tempestade de Idéias é uma técnica de trabalho em grupo onde todos contribuem livremente com idéias para a resolução de problemas.

No Quadro 1, a seguir, que apresenta a conclusão das discussões realizadas a partir das idéias dos enfermeiros sobre a assistência de enfermagem, observamos que o grupo tem como objetivo realizar uma assistência planejada, sistematizada e eficaz, fundamentada no aprendizado, no trabalho em equipe e em avaliações.

\section{QUADRO 1 - ASSISTÊNCIA DE ENFERMAGEM PRATICADA NO U.O.}

Individualizada.

Busca aprendizado para ser:

- sistematizada;

- eficaz;

- planejada.

Perspicaz enquanto está aberta para avaliação.

Fruto de um amadurecimento, de um trabalho em equipe, de uma relação empática.

Paciente é visto como um todo.

Apoiou-se em recursos humanos e materiais, falta o psicólogo.

Tem perseverança 
A finalidade desta assistência, assistir ao paciente como um todo e de forma individualizada, parece estar idealizada, considerando que os enfermeiros reconhecem a deficiência na assistência aos aspectos psicológicos. Os depoimentos dos enfermeiros revelam ainda uma falta de conhecimentos sobre a coleta de dados e uma resistência para registrá-los, quando afirmam:

- "O histórico tem ficado de fora, está sem vinculo com a prescrição".

- "Temos resistência em fazer o histórico pela falta de uso".

- "O histórico não é feito porque a enfermeira tenta resolver o imediato e o posterior fica para o futuro. Como a prescrição é para o momento, ele fica desvinculado".

Nas discussões sobre o processo de enfermagem praticado na U.Q. e nas conclusões apresentadas no Quadro 2 a seguir, o grupo relata que a maior parte dos dados não são registrados, mas que o processo de enfermagem começa com a avaliação do paciente. Nessa avaliação, são coletados dados para atender as situações imediatas.

\section{QUADRO 2 - PASSOS DO PROCESSO DE ENFERMAGEM PERCORRIDOS NA U.Q.}

\begin{tabular}{|c|c|}
\hline VISÍVEIS & INVISÍVEIS \\
(Registrados) & (Não registrados) \\
Coleta de dados & A maior parte dos dados não são registrados \\
& (Diagnósticos) \\
& (Objetivos) \\
Prescrição de enfermagem & (Implementação) \\
Evolução & (Avaliação) \\
\hline
\end{tabular}

VISÍVEIS (Registrados) Coleta de dados Prescrição de enfermagem Evolução INVISÍVEIS (Não registrados) A maior parte dos dados não são registrados (Diagnósticos) (Objetivos) (Implementação) (Avaliação)

Quando descrevem, passo por passo a prática do processo de enfermagem, os enfermeiros reconhecem que utilizam etapas que são registradas e outras que não são. Com base nessa descrição que partiu da própria experiência, entendemos que o grupo tem uma compreensão da estrutura do processo de enfermagem. Embora os passos do processo de enfermagem não tenham sido claramente definidos pelos enfermeiros, essa compreensão é um pouco chave, que fundamentou as discussões sobre a prática dessa metodologia de assistência na atividade seguinte.

Esse movimento foi fundamental para o desenvolvimento das atividades de ensino-aprendizagem subseqüentes.

$\mathrm{Na}$ atividade de ensino-aprendizagem I-03, fizemos uma análise dos registros do processo de enfermagem nos prontuários de pacientes internados na U.Q.. Essa atividade foi desenvolvida com base na compreensão da estrutura do processo de enfermagem e nas orientações fornecidas pelo coordenador. 
O desenvolvimento dessa atividade levou o grupo à observação da realidade de forma mais objetiva.

A análise das anotações de enfermagem nos prontuários possibilitou a confirmação da deficiência dos registros na coleta de dados e da dificuldade no levantamento e tratamento das necessidades psicossociais e psicoespirituais. Assim, pudemos compreender que essa assistência não atende o paciente como um todo.

No Quadro 3 observamos que os enfermeiros apresentam como os reais componentes do processo de enfermagem: coleta de dados, diagnóstico, determinação de objetivos, prescrição, implementação e avaliação.

\section{QUADRO 3 - CONCLUSÕES DA ANÁLISE DOS REGISTROS DE ENFERMAGEM NOS PRONTUÁRIOS DE PACIENTES DA U.Q. SOBRE OS COMPONENTES DO PROCESSO DE ENFERMAGEM}

\begin{tabular}{|c|c|c|c|}
\hline \multicolumn{2}{|c|}{$\begin{array}{l}\text { REAIS COMPONENTES DO } \\
\text { ROCESSO DE ENFERMAGEM }\end{array}$} & \multicolumn{2}{|c|}{ COMPONENTES DO PROCESSO DE } \\
\hline $\begin{array}{l}\text { Coleta } \\
\text { De } \\
\text { dados }\end{array}$ & $\begin{array}{c}\text { Informações } \\
\text { Dados } \\
\text { Análise } \\
\text { Priorização }\end{array}$ & Avaliação & $\begin{array}{l}\text { A maior parte } \\
\text { dos dados não } \\
\text { são registrados }\end{array}$ \\
\hline $\begin{array}{l}\text { Diagnóstico de } \\
\text { Objetivos } \\
\text { Prescrição de } \\
\text { Implementaçã } \\
\text { Avaliação } \\
\text { (Evolução de }\end{array}$ & $\begin{array}{l}\text { gem } \\
\text { em }\end{array}$ & $\begin{array}{l}\text { Prescrição não } \\
\text { (rotinas e cuida } \\
\text { Evolução incom }\end{array}$ & $\begin{array}{l}\text { dualizada } \\
\text { sicos) }\end{array}$ \\
\hline
\end{tabular}

A análise e priorização dos dados foram incluídas pelo grupo na fase de coleta de dados. A etapa de planejamento foi entendida como uma fase de determinação de objetivos e da prescrição de enfermagem. No Quadro 3, os enfermeiros estabelecem uma comparação entre os passos do processo de enfermagem e a forma como ele vem sendo desenvolvido na U.Q. Desse modo, o grupo concluiu que realiza uma avaliação incompleta e uma prescrição não individualizada em cuidados físicos e rotinas.

A coleta de dados perde a sua finalidade quando a etapa seguinte, o diagnóstico ou, pelo menos, um levantamento de problemas não for realizado. Como conseqüência da falta de registros da coleta de dados ou da não realização, todas as outras etapas ficam comprometidas. Nesse contexto, a prescrição de enfermagem surge como uma lista de rotinas a serem cumpridas.

No Quadro 4 a seguir, estão relacionados os determinantes das situações encontradas. 


\title{
QUADRO 4 - CONCLUSÕES DA ANÁLISE DOS REGISTROS DE ENFERMAGEM NOS PRONTUÁRIOS DE PACIENTES DA U.Q. SOBRE OS DETERMINANTES DA SITUAÇÃO ENCONTRADA.
}

\author{
Roteiro do histórico é ambíguo e confuso \\ Barreiras contra a história falta de estímulo para fazer histórico \\ Dificuldades para abordar o paciente \\ Faltam conhecimentos
}

A falta de conhecimento parece ser o principal determinante para a não aplicação de outras etapas do processo de enfermagem, principalmente da coleta de dados. Os outros determinantes apontados pelo grupo são decorrentes desse.

$\mathrm{Na}$ unidade I realizamos uma problematização da realidade. Assim, observamos e levantamos aspectos dessa realidade e selecionamos os fatores a serem considerados na solução dos problemas levantados.

Para FREIRE 6 partindo da compreensão da realidade, o homem "pode levantar hipóteses sobre o desafio dessa realidade e procurar soluções".

\subsubsection{Unidade II - Processo de enfermagem - Teorização}

A Unidade II compreendeu as seguintes atividades de ensino - aprendizagem: (II-1) aplicação da técnica de estudo dirigido - texto: "Processo de Enfermagem uma visão histórica" (texto-1) e discussão em pequenos grupos; (II-2) aplicação da técnica de estudo dirigido - texto: "Componentes do Processo de Enfermagem" (texto-2) e exposição dialogada; e (II-3) discussão conjunta com a presença de professor convidado.

Nessa Unidade, como sugerem DIAS BORDENAVE \& PEREIRA ${ }^{3}$, os pontoschave devem ser discutidos à luz da teoria e da pesquisa, buscando as soluções para o problema ou novos conhecimentos sobre o assunto em pauta. Essa etapa é chamada por esses autores de Teorização.

Nessa fase, que constituiu o momento mais abstrato do raciocínio, optamos por utilizar a técnica de estudo dirigido oferecendo material e tempo livre para a leitura. Em sala de aula, utilizamos a discussão em pequenos grupos, o registro das conclusões em quadros, a exposição dialogada, a discussão em comum e exercícios de aplicação prática.

O estudo dirigido foi uma atividade realizada pelos enfermeiros através de roteiros previamente preparados pelo coordenador. 


\section{Atividades de Ensino-Aprendizagem (II-1)}

O texto 1 - "O processo de Enfermagem - Uma Visão Histórica", consistia num resumo da evolução histórica do processo de enfermagem e foi fornecido aos enfermeiros no último dia de aula da Unidade I, quando fornecemos as instruções necessárias para a realização dessa atividade.

O estudo dirigido foi realizado por três enfermeiros em grupo e os demais preferiam a leitura individual. Ficamos disponíveis para o esclarecimento de qualquer dúvida, mas não fomos solicitados durante o período reservado para leitura.

A partir das conclusões elaboradas pelos enfermeiros nessa atividade e com base nos resultados obtidos na Unidade I, registradas em quadros, foram elaboradas conclusões sobre os determinantes da prática do processo de enfermagem na Unidade de Queimados, que estão apresentados na Quadro 5.

\section{QUADRO 5 - DETERMINANTES DO AVANÇO E DO ATRASO DO PROCESSO DE ENFERMAGEM NA UNIDADE DE QUEIMADOS}

\begin{tabular}{|c|c|}
\hline AVANÇO & ATRASO \\
\hline $\begin{array}{l}\text { Existência de políticas motivadoras no } \\
\text { serviço; }\end{array}$ & $\begin{array}{l}\text { Paciente não exige atendimentos } \\
\text { individualizados; }\end{array}$ \\
\hline Busca de valorização profissional; & Hegemonia médica; \\
\hline Avanço do trabalho em equipe; & História da enfermagem; \\
\hline $\begin{array}{l}\text { Disponibilidade de recursos humanos, } \\
\text { materiais e tecnológicos; }\end{array}$ & $\begin{array}{l}\text { Preocupação com o físico esquecendo } \\
\text { de ver o paciente como um todo; }\end{array}$ \\
\hline Filosofia comum de trabalho; & Desvalorização da classe; \\
\hline \multirow[t]{4}{*}{ Conhecimento, acesso a livros. } & Falta de conhecimento; \\
\hline & Bibliografia estrangeira; \\
\hline & Inconsciência entre teoria e prática; \\
\hline & Falta de hábito para leitura. \\
\hline
\end{tabular}

A partir dessas conclusões observamos que as instalações relacionadas à remuneração, horário de trabalho, sofrimento e agressividade dos pacientes abordadas na unidade anterior não foram apontadas pelos enfermeiros como determinantes do atraso do processo de enfermagem.

Essa fase de teorização teve continuidade com a realização de um estudo dirigido sobre os componentes do processo de enfermagem. Esse texto compreendeu um resumo sobre as etapas: coleta de dados, diagnóstico, planejamento, implementação e avaliação. Na realização dessa atividade os enfermos deveriam refletir sobre a importância e finalidade dos componentes do processo de enfermagem. 
Nessa fase os enfermeiros referiram dificuldades na compreensão dos nove padrões de respostas humanas e nos diferentes níveis de abstração na taxionomia de diagnósticos de enfermagem proposta pela NANDA.

Desse modo, realizamos uma exposição dialogada apresentando os pontos que consideramos importantes.

A atividade seguinte consistiu na realização de um exercício, segundo os passos do processo de enfermagem, a partir de dados apresentados pelo grupo, seguida de discussão conjunta com a presença de um professor convidado. $\mathrm{Na}$ compreensão da Taxionomia de diagnósticos de enfermagem.

Durante a realização do exercício, observamos que falta de dados sobre o paciente compromete todo o processo. Esse é um ponto importante considerando que a Unidade I foi observado que o grupo desconhecia a finalidade da coleta de dados.

A esse respeito, FERNANDES ${ }^{5}$ afirma que o uso de instrumentos de coleta de dados, ou sistema de registros inadequados dificulta o sucesso do emprego do processo de enfermagem. Quando muito longos, com perguntas ambíguas ou com dados que posteriormente não serão utilizados, levam à perda de tempo e frustrações.

A falta de utilização dos dados, já que os diagnósticos não tem sido firmados tem resultado na construção de instrumentos longos, repetitivos e ambíguos.

$\mathrm{Na}$ medida em que tentamos esclarecer os diagnósticos com base nas suas características definidoras e fatores relacionados, fomos percebendo quais os tipos de dados a serem coletados.

Durante a realização do exercício, a maior parte do tempo da aula foi utilizada para analisar os dados apresentados e firmar diagnósticos. As etapas de planejamento, implementação e avaliação foram pouco discutidas.

Pareceu-nos que nesse momento a preocupação do grupo estava voltada para as fases de coleta de dados e diagnóstico.

Verificamos que os enfermeiros não estavam habituados a pensar sobre os objetivos que pretendiam alcançar em cada ação que prescreviam. Como concluímos na Unidade I, a prescrição tem sido realizada sem vínculo com as outras fases do processo de enfermagem. Nesse contexto as ações não são prescritas de forma operacional. Em alguns casos, são prescritos os objetivos a serem alcançados, ou até instruções para que determinados materiais fiquem disponíveis para a implementação de uma ação que não está explicitada na prescrição.

Essa situação contribui para que o processo de enfermagem seja visualizado pelos enfermeiros como um processo burocrático e estático dissociado da prática.

\subsubsection{Unidade III - Elaboração de propostas para reformulação da Prática do Processo de Enfermagem na U.Q.}

Essa unidade abrangeu as seguintes atividades de ensino-aprendizagem: (III-1) aplicação da técnica de estudo dirigido "O possesso de enfermagem numa 
Unidade de Queimados: analise a proposta de reformulação segundo o modelo conceitual de HORTA $^{7}$ e discussão em pequenos grupos; (III-2) elaboração de propostas para a reformulação da pratica do processo de enfermagem na U.Q. e (III-3) avaliação.

Nessa unidade, pretendíamos dar continuidade à fase de teorização; deveríamos propor as hipóteses de solução e iniciar a fase de aplicação à realidade. Nesta etapa, utilizamos meios que favoreceram a discussão e o confronto do modelo ou pontos-chave com as conclusões da teorização.Esses confronto foi realizado através dos quadros com os registros das conclusões obtidas no levantamento dos pontos-chaves,e das conclusões obtidas na teorização.

Alem disso, os enfermeiros realizaram uma auto-avaliação quanto ao desempenho na fase de instrução e uma avaliação das experiências de aprendizagem.

A primeira atividade desenvolvida nessa unidade possibilitou uma revisão dos conceitos abordados na unidade anterior, agora aplicados ao modelo de HORTA.

Com a utilização da adaptação da Taxionomia I de diagnóstico de enfermagem proposta por ROSSI \& DALRI ${ }^{13}$, observamos que os enfermeiros encontravam os possíveis diagnósticos com maior facilidade, mas necessitavam confirmá-los com as características definidoras apresentadas na Taxionomia I proposta pela NANDA.

Esse exercício foi realizado em menor tempo (40 minutos) que o primeiro, já que as interrupções para esclarecimentos das dúvidas foram menos freqüentes.

O fato da U.Q. ser um serviço especializado facilita o reconhecimento de alguns diagnósticos, que com certeza irão se repetir como observaram os enfermeiros. A repetição dos diagnósticos diminuirá a necessidade de consulta às características definidoras. solução.

A partir da segunda atividade, os enfermeiros elaboraram as hipóteses de

Segundo DIAS BORDENAVE \& PEREIRA ${ }^{3}$, nessa etapa "os alunos propõem hipóteses de solução, as quais são confrontadas com os parâmetros da maquete do problema" ou ponto-chave.

Assim, os enfermeiros elaboraram propostas para reformulação da prática do processo de enfermagem. Na elaboração dessas propostas foram consideradas as discussões anteriores.

O registro das conclusões nos quadros possibilitou o confronto das propostas com os pontos-chave discutidos à luz da teoria.

As propostas de ROSSI \& DALRI ${ }^{13}$ foram apresentadas ao grupo para que fossem analisadas e modificadas. Desse modo, as propostas dos enfermeiros foram elaboradas a partir da análise dos estudos dessas autoras, à luz da teorização dos pontos-chave.

Durante a realização dessa atividade, os enfermeiros pareciam estar convencidos da importância da aplicação de todas as fases do processo. Assim, as soluções propostas apresentadas no Quadro 6, foram transformadas em planos de ação a serem aplicados na Unidade. 


\section{QUADRO 6 - PROPOSTAS DE REFORMULAÇÃO PRÁTICA DO PROCESSO DE ENFERMAGEM}

1. Coletar nas primeiras seis horas os dados relativos ao equilíbrio hemodinâmico, através de entrevista e exame físico;

2. O enfermeiro que interna o paciente fica responsável pelo preenchimento completo da coleta de dados, delegando para outros quando não for possível preenchê-lo. Isso deverá ocorrer em um prazo de cinco dias após a internação;

3. Fica estabelecido que serão firmados os diagnósticos de enfermagem nas primeiras seis horas. Serão escritas na folha de observação com data de avaliação, data de resolução e código de NANDA;

4. O objetivo para cada diagnóstico deverá ser estabelecido;

5. A prescrição deverá seguir o modelo adotado ${ }^{13}$;

6. A evolução deverá seguir o modelo adotado ${ }^{13}$;

Com a revisão de cada item do instrumento da coleta de dados, os enfermeiros puderam modificar termos que consideraram ambíguos ou repetitivos.

Dessa forma, entendemos que os instrumentos de coleta de dados devem ser periodicamente revisados pelo próprio grupo que o utiliza.

A priorização dos dados a serem coletados no momento da internação pode evitar a perda de tempo, e tranqüilizar o enfermeiro, que sente dificuldades em abordar o paciente nesse primeiro momento.

Para os enfermeiros acostumados a passar da coleta de dados para a prescrição, sem ao menos levantar problemas, o diagnóstico de enfermagem é um assunto novo e complexo. Detectamos, nos depoimentos levantados neste estudo, que os enfermeiros têm como proposta aplicar a fase de diagnóstico utilizando as categorias diagnósticas propostas pela NANDA, mas que reconhecem a necessidade de continuar estudando e discutindo, juntos, as dificuldades.

O grupo optou por estabelecer os objetivos na seqüência dos diagnósticos, mas sem o aprazamento. Os objetivos deveriam ser datados na medida em que fossem alcançados.

A aplicação dessas propostas no que se refere a fase de coleta de dados, diagnóstico e planejamento, refletirá nas fases seguintes determinando a necessidade de novas discussões.

$\mathrm{Na}$ última atividade dessa unidade, os enfermeiros deveriam fazer uma autoavaliação quanto ao seu desempenho durante a aplicação do plano de atividade educativa e quanto às experiências de ensino-aprendizagem vivenciadas.

Essa é uma forma de avaliação somativa, ou seja, é uma avaliação final do curso, realizada depois que vários objetivos tenham sido alcançados.

Realizamos também, uma avaliação formativa enquanto as atividades estavam sendo desenvolvidas, através da observação dos membros do grupo e, da análise dos depoimentos dos enfermeiros e dos registros das conclusões em quadros. 
Nesse esquema pedagógico, a avaliação como um processo contínuo é muito importante. Cada fase representa os objetivos a serem alcançados, e a passagem de uma fase para outra sem que isto tenha ocorrido pode comprometer o restante do processo.

Através dos depoimentos dos enfermeiros na auto avaliação, concluímos que eles estavam motivados para a implementação das propostas.

\subsection{Fase de Aplicação e Acompanhamento}

Essa etapa foi realizada na Unidade de Queimados com a aplicação de propostas elaboradas na fase de instrução. Foram realizadas reuniões utilizando-se a técnica de discussão em pequenos grupos, aproveitando as situações em que os enfermeiros estavam aplicando as propostas.

Após o término da fase de instrução, os enfermeiros providenciaram o impresso com o novo instrumento para a realização da coleta de dados e iniciaram a aplicação das propostas na internação do primeiro paciente. Durante um mês mais seis pacientes foram admitidos na Unidade. Acompanhamos a aplicação das propostas diariamente nos períodos da manhã e da tarde. Durante um período de 4 meses, transcorridos após a fase de Instrução, foram admitidos 13 pacientes na Unidade. O processo de enfermagem foi aplicado de acordo com as propostas elaboradas pelos enfermeiros em todos os pacientes.

Observamos que as maiores dificuldades estavam relacionadas à análise dos dados que levariam aos diagnósticos agrupados nas categorias necessidades psicossociais e psicoespirituais. Os enfermeiros referiram dificuldades, tantos na identificação dos diagnósticos desta área, como na prescrição das ações a serem implementadas. A evolução de enfermagem nem sempre fornecia dados para uma avaliação do paciente quanto ao alcance dos objetivos propostos. Alguns diagnósticos na forma como estavam descritos, levavam a diferentes interpretações.

Estas dificuldades foram discutidas em reuniões com os enfermeiros, quando foram elaboradas as seguintes propostas:

\section{QUADRO 7 - PROPOSTAS ELABORADAS NA FASE DE "APLICAÇÃO E ACOMPANHAMENTO"}

As categorias diagnósticas deverão ser vinculadas com os fatores relacionados e associadas às características definidoras dos diagnósticos; A prescrição e a evolução de enfermagem devem ser elaboradas nas seqüências dos objetivos;

Cada diagnóstico deve ser avaliado quanto ao alcance dos objetivos e esta avaliação deve estar registrada na evolução de enfermagem;

Deverá ser realizado um curso com base nas características definidoras dos diagnósticos relacionados às necessidades psicossociais e psicoespirituais, abordando também, as condutas de enfermagem para estes casos. 
As dificuldades apresentadas pelo grupo, ao analisar os dados que levariam aos diagnósticos agrupados nas categorias necessidades psicossociais e psicoespirituais podem estar relacionadas à falta de conhecimentos dos enfermeiros sobre o assunto, às próprias características do paciente nas primeiras semanas após a queimadura, e ao modelo de HORTA, adotado na U.Q. que prioriza as necessidades fisiológicas.

Durante a fase de Instrução, não era nosso objetivo, teorizar as dificuldades dos enfermeiros, relacionadas ao atendimento das necessidades psicossociais e psicoespirituais do paciente queimado. $\mathrm{Na}$ fase de Aplicação e Acompanhamento, essa questão novamente aparece como um fator que interfere na prática e que necessita uma solução. A proposta de realização de um curso sobre esse assunto poderá ser um meio para a solução desse problema.

Como dissermos, nessa levantamos questões que deverão ser abordadas posteriormente. As transformações da realidade criam uma nova situação que conterá novos problemas.

Para FREIRE ${ }^{6}$ "conhecer não é o ato através do qual um sujeito transformado em objetivo recebe dócil e passivamente os conteúdos que o outro lhe dá ou impõe. O conhecimento pelo contrário, exige uma presença curiosa do sujeito em face do mundo. Requer a sua ação transformadora sobre a realidade. Demanda uma busca constante. Implica em invenção e reinvenção".

Nos depoimentos dos enfermeiros na auto-avaliação, observamos que eles entendem a necessidade de continuar buscando, modificando a prática.

Essa busca deve ser contínua, não só porque não conseguimos esgotar o assunto, ms também porque como afirma FREIRE ${ }^{6}$, "todo saber traz em si a sua própria superação".

\section{CONCLUSÕES}

Este estudo nos permitiu concluir que:

- o plano de atividade educativa fundamentado no "Esquema do Arco" de MAGUEREZ ${ }^{8}$ possibilitou a análise e a reformulação da prática do processo de enfermagem na Unidade de Queimados;

- as fases do esquema pedagógico utilizado não são claramente delimitadas e estanques;

- o conhecimento da filosofia e objetivos do departamento de enfermagem, assim como da própria unidade, constitui um pré-requisito para a implementação do processo de enfermagem; 
- durante a fase de "observação da Realidade", a utilização da técnica Tempestade de Idéias não foi suficiente para que o grupo, partindo de uma visão idealizada, chegasse a uma visão objetiva da prática do processo de enfermagem. Essa percepção objetiva da realidade tornou-se possível com análise dos registros de enfermagem nos prontuários;

- na fase de "Teorização" foi necessária maior participação dos coordenadores (professor convidado e coordenador);

- o grupo de enfermeiros teve maior dificuldade na compreensão da "Taxionomia de diagnósticos de enfermagem";

- a implementação da fase de diagnóstico permitiu que os enfermeiros percebessem o vínculo entre essa e as outras etapas do processo de enfermagem, reforçando a importância da coleta de dados para que os diagnósticos possam ser firmados com segurança;

- os enfermeiros apresentam dificuldades em analisar os dados para afirmar diagnósticos e planejar as intervenções relacionadas aos aspectos psicossociais e psicoespirituais;

- a fase de evolução de enfermagem, como está sendo realizada, nem sempre oferece dados para uma avaliação do paciente quando ao alcance dos objetivos delineados no plano assistencial;

- a partir da aplicação das propostas delineadas nesse plano da atividade educativa criamos uma nova realidade, que já mostra a necessidade de outros estudos.

\section{NURSING PROCESS IN AN UNIT FOR BURNT PATIENTS; ANALYSIS AND REFORMULATION BASED ON THE PROBLEM RAISING PEDAGOGY}

The present study aims at elaborating and applying an education plan for nurses in a Unit for Burnt Patients, with the proposal of analyzing and reformulating the practice of the nursing process in this service.

The framework proposed by Charles MAGUEREZ ${ }^{8}$ made possible a substantial improvement in the nursing process in this service, and that this new reality claims for further studies.

UNITERMOS: nursing process, nursing education, nursing care 


\section{EL PROCESO DE ENFERMERÍA EN UNA UNIDAD DE QUEMADOS: ANÁLISIS Y REFORMULACIÓN FUNDAMENTADAS EN LA PEDAGOGÍA DE LA PROBLEMATIZACIÓN}

El presente estudio consistió en la elaboración y aplicación de un plan de actividades educativas para enfermeros de una Unidad de Quemados, con el objetivo de analizar y reformular la practica del proceso de enfermería en esa unidad.

El "Esquema del Arco" propuesto por Charles MAGUEREZ", fue adaptado como guía, el cual orientó en la escogencia y aplicación de las actividades de enseñanza-aprendizaje, implementados en este plan de actividades educativas.

Concluimos que este esquema pedagógico posibilitó el surgimiento de estas transformaciones en la práctica del proceso de enfermería en esta unidad, y que esta nueva realidad demuestra la necesidad de otras transformaciones.

UNITERMOS: proceso de enfermería, educación asistencia de enfermería

\section{REFERÊNCIAS BIBLIOGRÁFICAS}

01. CARVALHO. J. M. O processo didático. Rio de Janeiro: Fundação Getúlio Vargas, 1972.

02. DEWEY, J. Como pensamos. 3. ed. São Paulo: Nacional, 1959.

03. DIAZ BORDENAVE, J., PEREIRA, A. M. Estratégia de ensino-aprendizagem. 12 ed. Petrópolis: vozes, 1991.

04. FERNANDES, M.A. Processo de enfermagem: aplicação em uma Unidade de Hospital Escola do Interior. Rev. Esc. Enfermagem USP, São Paulo, n. 21, p. 88-99, 1987. Número especial.

05. FREIRE, P. Educação e mudança. 16 ed. Rio de Janeiro: Paz e Terra. 1990.

06. Extensão ou comunicação. 4ed. Rio de Janeiro: Paz e Terra. 1979.

07. HORTA, W. DA. Processo de enfermagem. São Paulo: EPU/EDUSP, 1979.

08. MAGUEREZ, C. Elementos para uma pedagogia de massa na assistência técnica agrícola. /Relatório apresentado à Coordenadoria de Assistência Técnica Integral da Secretaria da Agricultora do Estado de São Paulo. Campinas, 1970./ 
09. MARIA, V.L.R. et al. Implantação e desenvolvimento do processo de enfermagem num hospital especializado. In: CICLO DE DEBATES SOBRE ASSISTÊNCIA DE ENFERMAGEM, I São Paulo, 1988. Anais. São Paulo: Centro de Estudos de Enfermagem. p. 59-70.

10. NÉRICI, I.G. Metodologia do ensino: uma introdução. 2 ed. São Paulo: Atlas, 1981.

11. RONCA, A.C.C., ESCOBAR, V.F. Técnicas pedagógicas: domesticação ou desafio à participação. Petrópolis: Vozes, 1984.

12. SOUZA, M.F. de. Conhecimento e aplicação do processo de enfermagem entre enfermeiros formados no período de 1975 à 1979. São Paulo, p. 48. Dissertação (Mestrado) - Escola de Enfermagem, Universidade de São Paulo, 1981.

13. ROSSI, L.A., DALRI, M.C.B. Proposta para a reformulação da prática do processo de enfermagem junto ao modelo conceitual de horta, numa Unidade de Queimados./ Trabalho apresentado junto à Disciplina Processo de Enfermagem: análise e etapas operacionais, do Curso de Pós-Graduação da Escola de Enfermagem de Ribeirão Preto da Universidade de São Paulo, 1991.I

14. VILARINHO, L.R.G. Didática temas selecionados. 5 ed. Rio de Janeiro: Livros Técnicos e Científicos, 1984. 